\title{
Spatial Inequality Analysis of Fire Risk in China
}

\author{
Guohui Li, Lizeng Zhao, Weiping Han, Ying Wang \\ Tianjin Fire Research Institute of Ministry of Public Security, Tianjin, 300381, China \\ E-mail:liguohui@tfri.com.cn
}

Received 6 July 2016

Accepted 8 August 2016

\begin{abstract}
Gini coefficient and Lorenz curve are increasingly used to examine the inequality of wealth, income, economy, and health. The objective of the current study is to introduce Gini coefficient and Lorenz curve, coefficient of variation (CV), and spatial equilibrium index (SEI) to explore the spatial inequality and spatial-temporal evolution of fires in China based on the fire statistics from 2003 to 2012. The spatial inequality of fire risk is influenced by population, provinces, regions, and fire causes. The results indicate that the spatial inequality of the fire deaths tends to become more intense over time, and shows spatial concentration. However, the number of fires becomes more decentralized distribution. As regards the three regions, the inequality of the number of fires in the central region (with Gini $>$ 0.4 ) is relatively obvious. As regards the seven causes, playing with fire, smoking, and improperly using fire presents extreme inequality in different provinces. The provinces with a high level of fire risk for different factors are depicted in maps by using ArcGIS, which can explicitly illustrate the spatial distribution characteristics of fire risk. The conclusions also demonstrate that $\mathrm{CV}, \mathrm{SEI}$, and Gini are suitable for analysis of the spatial inequality of fire risk.
\end{abstract}

Keywords: Spatial inequality; Fire risk; Gini coefficient; Coefficient of variation; Spatial equilibrium index

\section{Introduction}

The inequality in income and wealth have attracted tremendous attention and been widely studied. However, little effort has been paid to the spatial inequality of fire risk. The fires, one of the most critical issues, are related to the society stability and public safety. In this paper, the fire risk refers to the number of fires and the casualties. The more the number of fires or casualties are, the higher the fire risk. The fire risks are different in the geographic distribution, which makes it difficult for the fire departments to make effective control policies. Meanwhile, the fire resources in China are limited. The inequality phenomenon is ubiquitous in nature. Similar to the income inequality, there is also a spatial inequality for the fire resources. The fire departments should assign the limited fire resources scientifically and rationally, and make the specific firefighting measures. Furthermore, the spatial distribution of fire risk needs different measures. For optimizing fire-fighting and rescue forces, the placement of city fire risk and the fire rescue services based on the spatial distribution of urban fire risk should be obtained. Therefore, it is necessary to study the spatial inequality of the fire risk.

The city fires are influenced by socio-economic development, living conditions, and climate factors ${ }^{1}$. However, these factors show obvious spatial heterogeneity, and the fires will also be associated with the geographical factors. The economic development of China is uneven, and there are large gaps between the eastern and western regions. Yang et al. discussed the relationship between the fires and the socio-economic factors from the perspective of provinces in China. They found that the number of fires tend to increase with the economic development ${ }^{2}$. Many scholars have discovered that the temperature and humidity changes have great influence upon fire risk $^{3-5}$. Because of Chinese climate difference in north and south, there is a distinction between the fires in north and south China. 
The statistics indicate that the number of fires and the fire deaths are different in each province. In 20032012, China responded to an average of 142,124 fires per year, which caused 1,688 deaths and 1,367 injures annually. The number of fires varies greatly in different provinces. For example, Shandong accounted for $7.8 \%$ of the reported fires in 2012, and Henan only 3.36\%. The fatalities of Guangdong are forty-three times as many as that of Jilin ${ }^{6}$. The reasons for this phenomenon are mainly due to the population factors and the economic factors. Large number of population, production enterprises and fire hazard places result in the high fire risk.

The fire resources and the professional firefighters in China are insufficient, and it is useful to assess the spatial differences of fire risk in fire supervision and management. The fire-fighting measures largely depend on the fires' characteristics, such as the spatial distribution, human factors, and climate. Based on the fire statistics, the research studies of spatial inequality and variation have been carried out. However, most of the spatial-temporal distribution studies focused on the forest and grassland fires ${ }^{7-9}$. There have been few studies on the spatial-temporal feature of the city fires. $\mathrm{Lu}$ et al. discussed the associations between the deaths of high-casualty fire and the spatial-temporal factors in China $^{10}$. They obtained the key provinces correlating with high levels of deaths or losses. The results indicate that Beijing, Shandong and Jilin are strongly associated with fatality level $\geq 10$. Most of the coastal provinces tend to be related with electrical fires, but the northwest and northeast provinces tend to be associated with smoking, playing with fire, and improperly using fire ${ }^{11}$. Yang and Zhou discussed the fire situation and the fire characteristic of China ${ }^{12}$. They proposed some key lessons that should be learned from the fire cases: how to alert the sleeping people by using the high-quality equipment of fire detection and alarm, how to make full use of the equipment in bad weather, and how to take emergency measures.

The spatial inequality can also be known as spatial aggregation and hotspots. The idea of the spatial aggregation has been applied in terrorist attacks ${ }^{13}$, and crime analyses ${ }^{14}$. The spatial characteristics of city fires are usually studied without considering the inequality and the variation. The literature investigation has shown that the spatial aggregation and hotspots of city fires still remain undetected. There are nearly no previous research studies that use ArcGIS, Gini coefficients, and Lorenz curve to analyze the spatial inequality of city fires. To explore the spatial distribution pattern of city fires, this work adopts Gini and ArcGIS. As a classical approach, Gini coefficient and Lorenz curve have been widely used to measure the distribution of wealth, income, biomedicine, and the selectivity of kinase inhibitors ${ }^{15-17}$. The fire risk has strong region characteristic $^{18}$. By providing a measure of inequality, the Gini coefficient can identify how the level of inequality is, and the Lorenz curve can explore which province has the highest fire risk $^{19}$. It will reveal the driving factors of the spatial inequality of fire risk by further study on the correlation analysis between the socio-economic factor and Gini coefficients ${ }^{20}$.

The aim of this study is to measure the inequality of fire risk in China based on the fire statistics from 2003 to 2012. First, we seek to identify the spatial inequality of the fire deaths and the number of fires at province level. Second, according to the level of economic development and the administrative division of China, the 31 provinces are divided into three economical belts: the eastern region, the central region, and the western region $^{21}$. The spatial inequality of fire risk between the three regions is investigated. Finally, the spatial distribution characteristics of different fire causes are explored. The results of the spatial inequality of fire risk will provide feasible countermeasures for the fire control strategies.

\section{Methods and Data}

\subsection{Data}

The analytical data are collected from the Fire Service Bureau in Ministry of Public Security of China ${ }^{6}$. The number of fires and the fire deaths in each province are available. Seven fire causes are distinguished, including arson, electricity, disregarding safety rules, improperly using fire, smoking, playing with fire, and spontaneous combustion (the fire causes of lightening, static, unknown and others are excluded).

According to the statistics from 2003 to 2012, the average annual number of fires and the fire deaths are 139,839 and 1,689. In Fig. 1, the fire deaths decrease quite steadily after 2005, while the number of fires fluctuates significantly. The number of fires increases slightly during 2003-2005. From 2006 to 2011, the 
number of fires decreases from 140,672 in 2006 to 125,417 in 2011 for a decrease of $12.4 \%$ except for 2007 when 163,521 fires occur, then increasing $21.3 \%$ to 152,157 in 2012 .

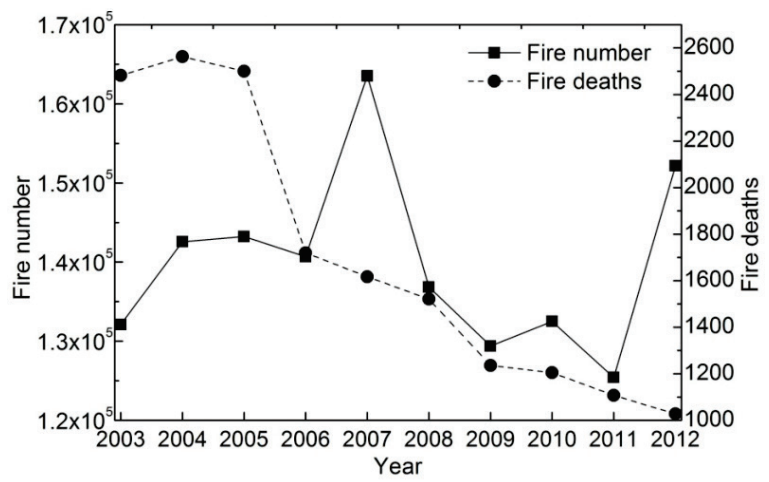

Fig. 1. Number of fires and deaths in China

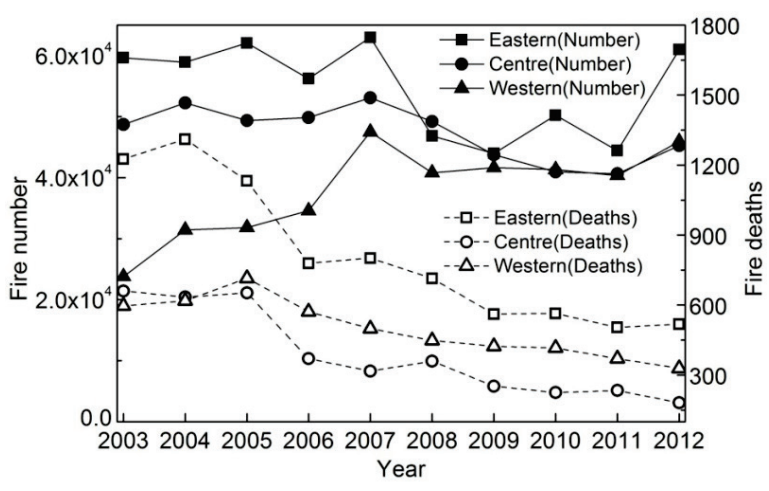

Fig. 2. Number of fires and deaths by regions

The economic development and living conditions in each province is different. In order to differentiate between regions of high and low inequality of fire risk, 31 provinces in China are divided into three regions: eastern region, central region, and western region. The eastern region includes eleven provinces: Beijing, Tianjin, Hebei, Liaoning, Shanghai, Jiangsu, Zhejiang, Fujian, Guangdong, Shandong, and Hainan. The central region includes eight provinces: Shanxi, Jilin, Heilongjiang, Anhui, Jiangxi, Henan, Hubei, and Hunan. The western region includes twelve provinces: Neimenggu, Guangxi, Chongqing, Sichuan, Guizhou, Yunnan, Xizang, Shanxi, Gansu, Qinghai, Ningxia, and Xinjiang. Because of the lack of fire statistics, the data of Taiwan, Hong Kong, and Macao are excluded. Before 2006, there is significant difference for the number of fires among the three regions as shown in Fig. 2. The eastern region accounts for the most fires and deaths. The number of fires in the western region is relatively less, and the number of fires is closed to that of the central region in 2008-2011. It should be noted that the number of fires in the western region increased $93.2 \%$ during the period of $2003-2012$ to 45,925 by the end of 2012, and the number of fires is more than that in the central region. The results may be influenced by the rapid development of society in the western region, including the frequent economic activity and population movements. As regard the deaths, the phenomenon is different from the number of fires. The deaths in the eastern region, the western region, and the central region are in order of decreasing frequency. The deaths in the western region are more than that in the central region. The results also suggest that the social economy in the western region is quite active.

The occurrence of fires is closely related to human activities, and the population factor should be considered $^{22}$. Fig. 3 shows the changing trend of the number of fires and the fire deaths per million people in the three regions. Compared with Fig. 2, there are a few changes. First, the number of fires in the western region is higher than that in the other two regions after 2008, and the value in the eastern region becomes the smallest. The other change is that the fire deaths in the western region are close to that in the eastern region. The results indicate that, the fire situation in the western region tend to be more severe.

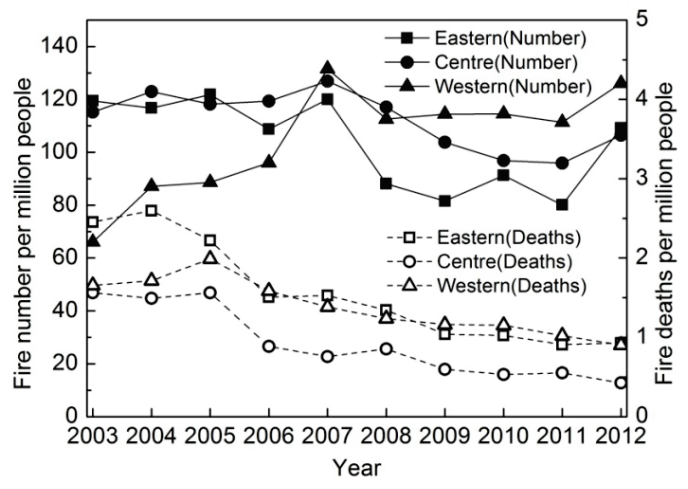

Fig. 3. Number of fires and deaths per million people

The number of fires caused by different causes is depicted in Fig. 4. Looking at the trends from 2003 to 2012, several observations are worth attention. First, most of the fires are caused by electricity and improperly using fire, which account for nearly half of the total fires. The electricity fires keep growth trend over the time. The wide use of large number of electrical equipment may contribute to the results. Secondly, there is a steady growth trend for spontaneous combustion fires, which may be caused by the climate 
change, and the growth of car spontaneous combustion accidents. Thirdly, the improperly using fire and arson continuously decrease except for 2012 when a slight rise occurred. Actually, most of the fires relate to human behavior and human errors ${ }^{12}$. The different human habits and living conditions in each province may lead to the spatial inequality of fire risk.

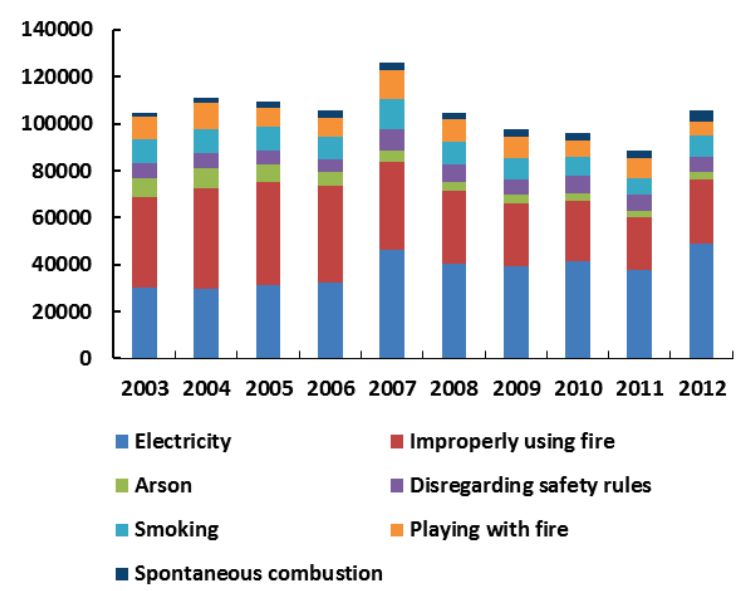

Fig. 4. Number of fires of different causes

\subsection{Methods}

\subsubsection{Gini coefficient and Lorenz curve}

The inequality of fire risk can be measured by using Gini coefficient. Gini coefficient is the most popular measures of inequality ${ }^{23-25}$. It has been widely used by economists to measure the income inequality ${ }^{26-27}$. The Gini coefficient has its own advantage than other methods because it is independent of the absolute values and compares each value not to the mean but to every other value $^{28}$. Furthermore, Gini coefficient is a novel development, and can measure inequality between different geographical regions. The results are particular relevance to the fire policymakers ${ }^{29}$. The results of Gini coefficients and Lorenz curve reflect the overall characteristics of the research space. The results are especially suitable for the decision-making departments.

Gini coefficient can be expressed by Lorenz curve as shown in Fig. 5. The absolute equality curve denotes an absolute equality of fire risk in space. The area between the absolute equality curve and Lorenz curve is $\mathrm{A}$, and the area under the Lorenz curve is B. The Gini coefficient is equal to $\mathrm{A} /(\mathrm{A}+\mathrm{B})$. The Gini coefficient is generally solved by the following integral formula.

$$
G=1-2 \int_{0}^{1} L d x
$$

In order to simplify the calculative process, Gini coefficient is calculated $\mathrm{as}^{24}$ :

$$
\text { Gini }=\sum_{i=1}^{n} P_{i} F_{i}+2 \sum_{i=1}^{n-1} P_{i}\left(1-C_{i}\right)-1
$$

where $P_{i}$ represent the proportion of the number of population or provinces, $F_{i}$ are the proportion of the fires or the deaths, and $C_{i}$ are the cumulative distribution of fires or deaths. A Gini coefficient of 0 indicates perfect equity, and 1 indicates absolute inequality. Generally, the Gini coefficient of 0.4 is seen as the warning line for the gap between rich and poor ${ }^{30}$.

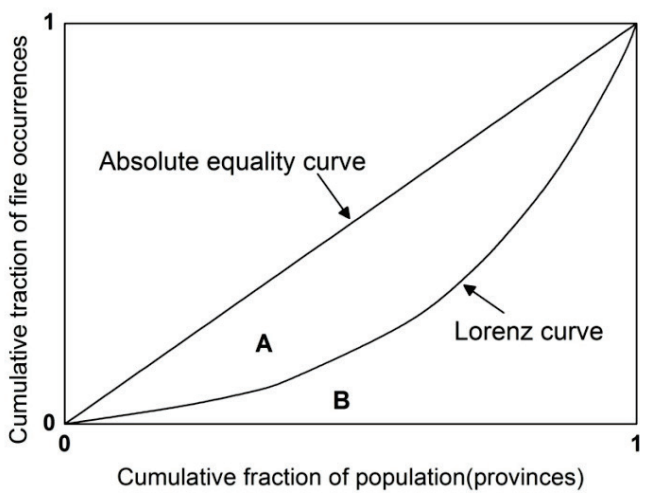

Fig. 5. Gini coefficient and Lorenz curve

\subsubsection{Coefficient of variation}

To avoid one-sidedness of the single method, coefficient of variation (CV) and spatial equilibrium index (SEI) are introduced to measure the spatial inequality of fire risk.

$\mathrm{CV}$ can be used to reflect the stability, consistency, and spatial differences between the provinces. $C V$ is calculated as:

$$
C V=S / \bar{X} \times 100 \%
$$

where $S$ denotes the standard deviation, and denotes the mean value. A greater value of $C V$ indicates more diversity and more inconsistency. On the contrary, a smaller value of $C V$ indicates more homogeneous distribution of fire risk.

\subsubsection{Spatial equilibrium index}

SEI is established to illustrate the relative fire risk by considering the population factor. The SEI of each province is given by the formula: 


$$
S E I_{i}=\frac{f_{i} / F}{p_{i} / P}
$$

where $f_{i}$ and $\mathrm{F}$ are the number of fires in the $i$-th province and the overall national number of fires, respectively. $p_{i}$ and $P$ are the number of population in the $i$-th province and the overall national population, respectively.

If the SEI of some province is more than 1, the fire proportion of fire occurrences in this province is larger than the proportion of the population, and the fire risk of the province is relatively serious. Conversely, the province has a lower fire risk.

\section{Results and Discussion}

\subsection{Coefficient of variation analysis of fire risk}

Based on the equation (3), the $\mathrm{CV}$ of the number of fires and the fire deaths in 2003-2012 are calculated as shown in Fig. 6. Firstly, the CV is calculated based on 31 provinces, and the sample values are the number of fires and the deaths in each province. Secondly, the CV is calculated based on the population, and the sample values are the number of fires and the deaths per million populations in each province.

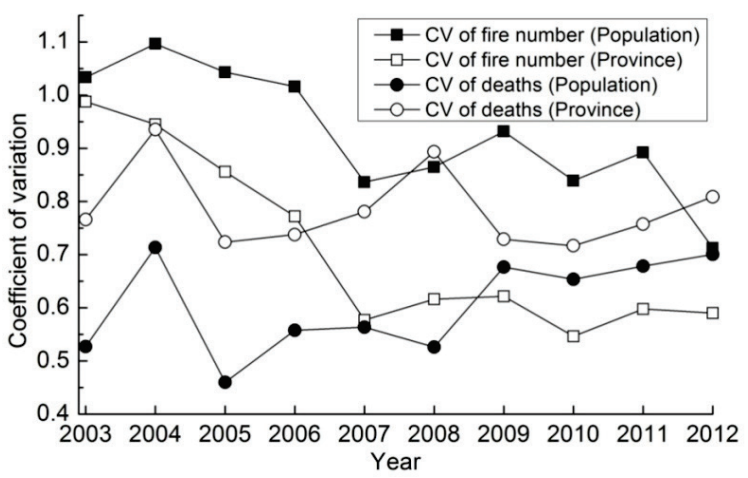

Fig. 6 . The change of $\mathrm{CV}$ over time

The annual CV values show fluctuating changes. The spatial difference of the number of fires per million populations is more obvious than that of only considering provinces. The former can better reflect the actual situation, because the fire risk has close relationship with the human activities. However, the spatial difference of the fire deaths based on province is more obvious than that of the deaths per million populations.
From 2004 to 2007, both of the spatial differences of the number of fires show an obviously decrease with the $\mathrm{CV}$ of province from 0.945 to 0.577 and the $\mathrm{CV}$ of population from 1.096 to 0.837 . During the period of 2007-2012, there is no obvious change for the spatial differences of the number of fires except for 2012 when the spatial differences of the number of fires per million populations decreased rapidly. There are two stages for the spatial differences of the number of fires base on province: the reduce stage from 2003 to 2007, and the steady stage from 2007 to 2012. This indicates that the spatial different of the number of fires tends to be weaken.

\subsection{Spatial inequality analysis of fire risk}

\subsubsection{The overall spatial inequality}

In this paper, we are primarily concerned with the inequality of fire risk from the perspective of province area. The spatial inequality can be explored by the Gini coefficients and Lorenz curve. Because of the different socio-economic factors, each province may present different levels of fire risk. Just as the wealth inequality, the spatial distribution of fire risk in China also shows inequality. Fig. 7 and Fig. 8 depict the Lorenz curves and Gini coefficients for 31 provinces in China from 2003 to 2012. Based on the international standard for interval division, the Gini coefficients are divided into three intervals: when $\mathrm{G}<0.2$ there is no spatial inequality for fire risk, meaning that the fire risk in each province is similar, $0.2 \leq \mathrm{G} \leq 0.4$ denotes a major spatial inequality of fire risk, and $0.4>\mathrm{G}$ indicates an extreme inequality of fire risk among the 31 provinces.
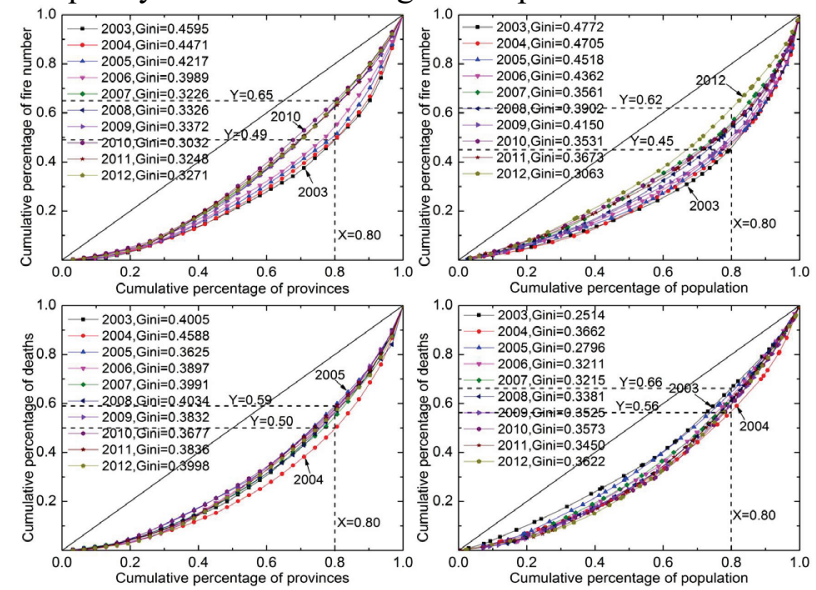

Fig. 7. Gini coefficients and Lorenz curves by provinces 
The tendency of the Lorenz curves with time for the number of fires is more obvious than that of the fire deaths as shown in Fig. 7. The number of fires and the fire deaths produced by the top $20 \%$ of provinces can be identified based on the Lorenz curve in Fig. 7. With regard to the number of fires, the top $20 \%$ of provinces account for $35 \%$ of the total fires in 2010 and increased to $51 \%$ in 2003 , the top $20 \%$ of population account for $38 \%$ of the total fires in 2012 and increased to $55 \%$ in 2003. For fire deaths, the top $20 \%$ of provinces account for $41 \%$ of the total deaths in 2005 and $50 \%$ in 2004 , the top $20 \%$ population account for $34 \%$ of the total deaths in 2003 and $44 \%$ in 2004. It indicates that the spatial inequality phenomenon of fire risk began to get better.

The spatial inequality of fire risk is changing over time. In Fig. 8, all of the Gini coefficients are greater than 0.2 , meaning that the fire risk presents spatial inequality. With regard to the number of fires, the spatial inequality of unit population is stronger than that of only considering provinces. It illustrates that the influence of the population factors on the fire distribution is more obvious. In 2003-2006, both of the Gini coefficients of the number of fires are close or more than 0.4 , indicating an extreme spatial inequality. In 2003-2006, nearly half of the fires occurred in only $20 \%$ of the provinces (about six provinces). After 2007, the Gini coefficients of the number of fires are lower than 0.4 except for 2009, and the fire risk tend to be less spatial inequality.

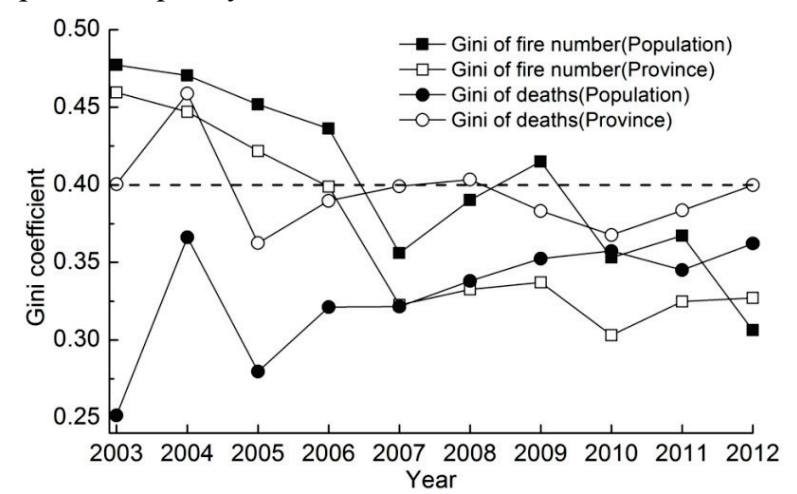

Fig. 8 . The spatial inequality of the number of fires and deaths

The results of the fire deaths show an opposite tendency. The spatial inequality of the fire deaths of unit population is weaker than that of only considering provinces. Simultaneously, the Gini coefficients for the fire deaths of unit population vary from a low of 0.251 in 2003 to a high of 0.362 in 2012, indicating that the spatial inequality phenomenon of the fire deaths becomes more obvious over time. Because of the high value of the Gini coefficients (most of the Gini coefficients exceed 0.35 over the ten years), the distribution of the fire deaths of provinces keeps spatial inequality over time. The results indicate that more and more fire deaths are produced by only few provinces, and the fire deaths tend to be spatial aggregation.

\subsubsection{Spatial inequality of regions}

To identify if the national inequality of fire risk is caused by the regional inequality, the spatial inequality per million populations within three regions is respectively studied as shown in Fig. 9. The spatial inequality of the number of fires within the western region is more obvious than that in the other two regions after 2006. The Gini coefficients of the number of fires are more than 0.4 from 2003 to 2012. In 2003-2006, the Gini coefficients of the number of fires within the central region are more than 0.45 , and the spatial inequality is the most obvious. However, the Gini coefficients of the number of fires in the central region decreases sharply in 2007 , then a slightly increasing until 2009, and declining over the last three years to 0.250 in 2012. The Gini coefficients of the eastern region decline fairly obviously, a $52 \%$ decrease over the ten years. Overall, the number of fires in the western region exhibits a more obvious spatial aggregation, which means that most of the fires are concentrated in the few special provinces. However, the spatial inequality of the number of fires in the central and eastern regions become less obvious, which means that the number of fires in these provinces don't appear to be much different.

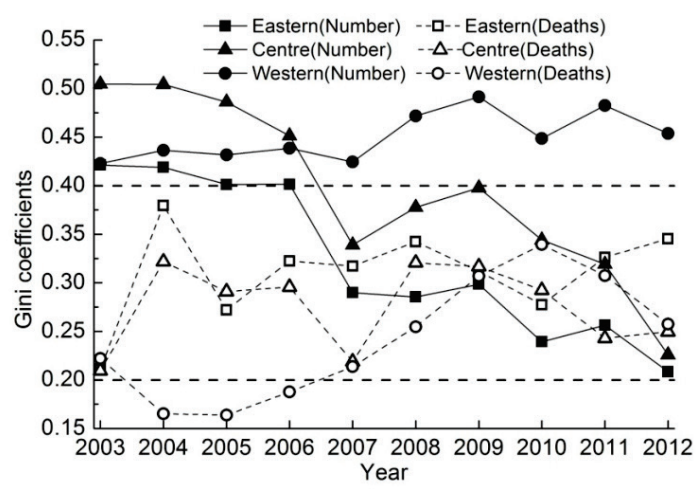

Fig. 9. The internal inequality in the three regions

With regard to the deaths, the spatial inequality is less obvious than that of the number of fires. Except for the high inequality level in 2004 and the low inequality level in 2003, there is a slight growth trend for the inequality phenomenon of the deaths within the eastern 
region. The Gini coefficients of the deaths within the central region show a cyclical change of five years. The most obvious characteristics are the straight-line growth of the western region from 2005 to 2010 , and then decreasing until to 2012. The spatial inequality in the western region achieves the maximum degree in 2010 and then begins to weaken in 2011-2012. The results indicate a significant feature that the spatial inequality within the eastern and central regions tends to become lower, while the western region becomes higher.

The changing in the western region should be noted. With the rapid development of economy and society in the western region, the fire situation becomes worsen. The development of economy in each province exist large gap, and therefore the fire risk between different provinces is obviously different. It can be found that Ningxia, Xinjiang, Neimenggu, and Qinghai account for the highest number of fires per million populations. The fire department should further increase the fire resources in the western provinces.

\subsubsection{Spatial inequality of fire causes}

To make national fire protection policy, the fire departments should grasp the overall distribution characteristics of the fire situation. Furthermore, they should also understand the national spatial distribution characteristics of the fire causes, because different fire causes need different countermeasures. Li et al. have explored the relationship between the fire causes and the influencing factors ${ }^{11}$. They identified that different types of fires present a certain spatial distribution pattern in the 31 provinces.

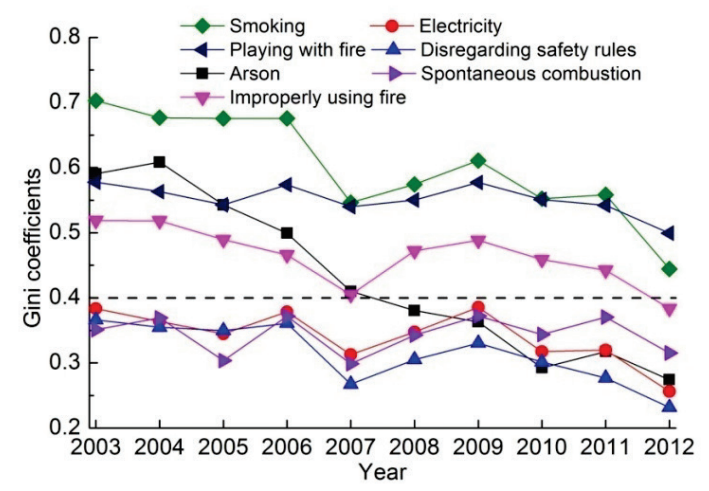

Fig. 10. The spatial inequality by fire causes

The fire causes are divided into 11 categories in China. Because of the lack of data or too little, only seven categories with the most number of fires are considered as shown in Fig. 10. There is no accurate statistics for the fire deaths of different causes. Therefore, only the number of fires is analyzed. Several crucial findings are explored as shown in Fig. 10.

First, the level of spatial inequality for the seven causes appears to be different. The fire causes can be classified into three categories according to the spatial inequality level: the high level of inequality, including smoking, playing with fire, and improperly using fire; the low level of inequality, including electricity, disregarding safety rules, and spontaneous combustion; and the medium level, including arson. With regard as the high inequality causes, the fire risk tends to be concentrated in the specific provinces, and the fire highrisk provinces should be given certain inclination in policy. As for the low inequality causes, the fire risk tend to be widely distributed, and each province should strengthen management. For example, Gini coefficients of the electrical fires are less than 0.4 , and the spatial inequality is relatively small. However, the electrical fires in each province are the most.

Secondly, with the highest Gini coefficients, the smoking and playing with fire present extreme spatial inequality. The Gini coefficients of smoking are more than 0.65 in 2003-2006, indicating a distinct spatial aggregation. The Gini of smoking is 0.703 in 2003, and most of the smoking fires are concentrated in Jilin, Shanghai, Ningxia, and Beijing. The four provinces with $8.1 \%$ of the total population account for $50.9 \%$ of the total number of smoking fires in 2003. Even the Gini coefficients of smoking decline to the smallest 0.444 in 2012, the top ten provinces (Ningxia, Neimenggu, Qinghai, Xinjiang, Heilongjiang, Jilin, Tianjin, Liaoning, Shanxi, and Gansu) with $18.3 \%$ of the population still account for $48.6 \%$ of the total fires. During the ten years, Shanghai shows the most significant changes. The number of smoking fires per million populations changed from 38.9 in 2003 to 5.8 in 2012. This may be related to the highly developed firefighting construction and the high fire safety awareness, which needs further study. The spatial distribution characteristics of playing with fire (with Gini $>0.50$ ) are also very obvious. The top eight provinces, Ningxia, Jilin, Qinghai, Liaoning, Beijing, Neimenggu, Heilongiiang, and Shanghai, with $13.6 \%$ of the total population, account for more than half of the playing with fire in 2003. In 2012, the top eight provinces are Ningxia, Qinghai, Neimenggu, Xinjiang, Liaoning, Gansu, Xizang, and Jilin. It is obvious that most of the 
two types of fire are concentrated in northeast and northwest provinces.

Thirdly, the overall changing trend seems to be weakened over time, and all the Gini coefficients decrease in fluctuation over time. The results indicate that the spatial inequality begins to weaken, especially the arson, with Gini coefficients ranging from 0.591 in 2003 to 0.274 in 2012 . The top ten provinces with $21.2 \%$ of the population account for $68.5 \%$ of the total arson fires in 2003 , while $24.1 \%$ of the population account for only $43.1 \%$ of the total fires in 2012 . As for arson fires both in 2003 and in 2012, the top ten provinces include Liaoning, Jilin, Heilongjiang, Ningxia, Xinjiang, Qinghai, Shandong, and Beijing, which are the hot spots of the arson fires. Another critical issue is that: the Gini coefficients of the seven causes reached the minimum in 2012, but the number of fire increased abruptly. This forebodes that the number of fires in each province tends to increase, and the fire situation becomes more serious in the whole country.

\subsection{Fire spatial equilibrium index}

The above analysis mainly focused on the spatial inequality of fire risk. CV and Gini can reflect the overall spatial inequality, but cannot visually identify which province accounts for the highest risk of fire. To explore the specific fire risk in each province, the SEI is calculated as shown in Fig. 11 and Fig. 12. With regard to the fire deaths, there are 16-17 provinces with SEI $>1$ in 2003-2012, except for 2004 when only 14 provinces. For the number of fires, there are 14-17 provinces with SEI $>1$. The province with a value of SEI $>1$ indicates that the fire risk is higher than the national average fire risk, and the fire situation is fairly severe. On the contrary, the provinces with $\mathrm{SEI}<1$ represent the relatively lower fire risk.

In Fig. 11 and Fig. 12, the spatial difference of the number of fires is more serious than that of the fire deaths. In 2003-2012, the maximum value of SEI for the number of fires reaches 6.53 in Jilin, and the proportion of fire is nearly six times more than the proportion of population. By contrast, the maximum value of SEI for the fire deaths is only 3.99 in Xizang.

With regard to the fire deaths, Beijing, Tianjin, Shanghai, Zhejiang, Fujian, Chongqing, Guangdong, Guizhou, Yunnan, Neimenggu, Xizang and Xinjiang have been maintained at the high risk level over the ten years. Hebei, Anhui, Jiangxi, Shandong, Henan, Hubei, Shanxi(2), and Gansu with SEI of less than 1, show the low fire death risk. It should be noted that the per capital deaths in Xizang is much higher than the average. The SEI of Ningxia is more than 3 during the whole period, indicating a very serious fire risk.

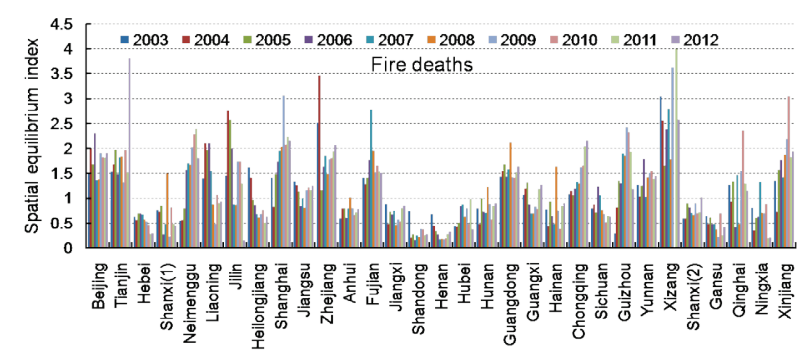

Fig. 11. SEI of the fire deaths

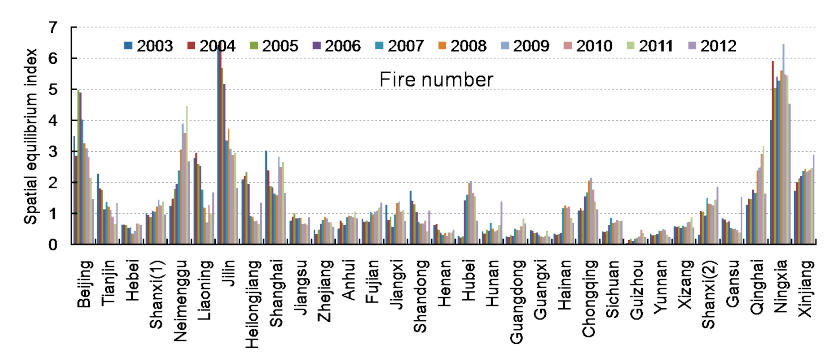

Fig. 12. SEI of the number of fires

With regard to the number of fires, SEI vary from the lowest of 0.03 in Guizhou to the highest of 6.53 in Jilin, meaning the huge spatial different. The risk of the number of fires in Beijing, Neimengu, Jilin, Shanghai, Ningxia, and Xinjiang is much higher than the average. The per capita fire in Ningxia is more than the other provinces, and the underlying reasons are still unknown. Additional research studies need to be carried out.

Based on the calculation values, SEI is divided into five levels, and the provinces with different SEI are depicted in the form of maps by using ArcGIS. The results of the latest four years (2009-2012) are shown in Fig. 13 and Fig. 14. It is clear that the fire situation tend to be spatial aggregation. That is, most of the fires and deaths are concentrated in only a few provinces. The high risk level of the fire deaths per capital are mainly distributed in the southeast and western regions, including Xizang, Xinjiang, Qinghai, Neimenggu, Chongqing, Yunnan, Guizhou, Guangdong, Fujian, Zhejiang, Jiangsu, Shanghai, Beijing, and Tianjin. The fire risk of most central provinces is lower than the average. Unlike the fire deaths, the high risk of the number of fires are mainly distributed in the western, northeast, and central regions, such as Liaoning, Jilin, Ningxia, Neimenggu, Xinjiang, Qinghai, Shanxi, Hubei, 
Chongqing, and Jiangxi. The developed municipalities, such as Beijing, Tianjin and Shanghai, show high risk for both of the number of fires and the fire deaths.

It should also be noted that Xizang and Shanghai maintained the highest risk level of the fire deaths during the whole period, and Beijing, Neimenggu, Jilin, and Ningxia always stay in high risk levels of the number of fires from 2009 to 2012. It can be summarized that the high fire risk regions will not change significantly over time.

An interesting phenomenon is that the high fire mortality may not be associated with the high occurrence rates, such as Xizang and Ningxia. The socio-economic conditions, under which it occurs, are different. Therefore, different countermeasures should be taken to deal with the fire risk.

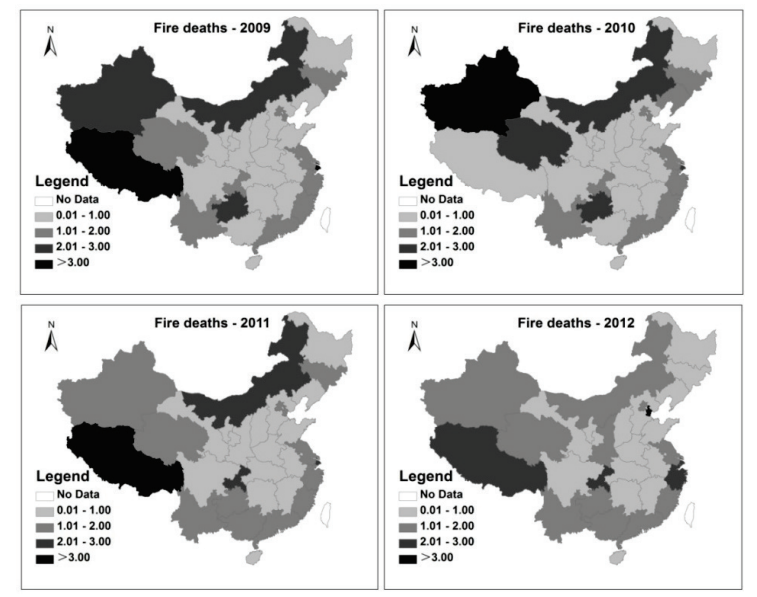

Fig. 13. Spatial distribution maps of SEI for fire deaths

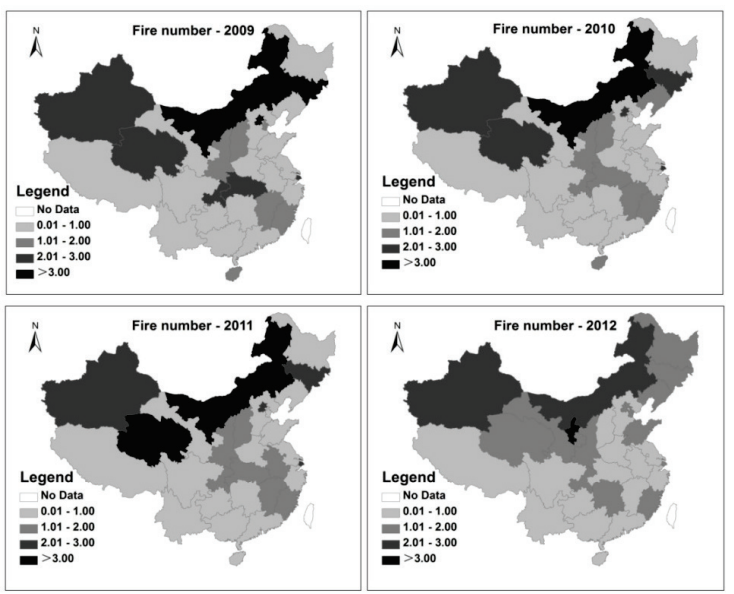

Fig. 14. Spatial distribution maps of SEI for fire number

It should also be noted that Xizang and Shanghai maintained the highest risk level of the fire deaths during the whole period, and Beijing, Neimenggu, Jilin, and Ningxia always stay in high risk levels of the number of fires. An interesting phenomenon is that the high fire mortality may not be associated with the high occurrence rates, such as Xizang and Ningxia. The socio-economic conditions, under which it occurs, are different. Therefore, different countermeasures should be taken to deal with the fire risk.

Smoking, playing with fire, and improperly using fire have shown extreme spatial inequality as depicted in Fig. 10. These fires tend to be spatial aggregation at provincial level, and the hot spots can be explored according to SEI. The spatial distribution of different fire causes are identified based on the value of SEI, and the spatial distribution maps of fire causes in 2012 are depicted in Fig. 15.

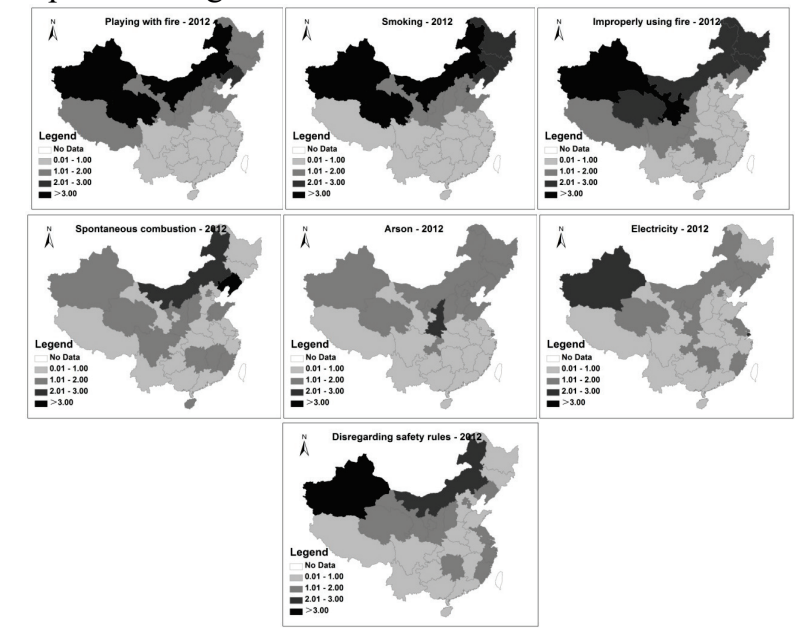

Fig. 15. Spatial distribution map of SEI for fire causes in 2012

The SEI of smoking and playing with fire vary widely, especially the playing with fire (SEI ranges from 16.3 to 0.05 ). A larger Gini coefficient means a more significant inequality. In 2012, playing with fire, smoking, and improperly using fire represents significant spatial aggregation, which can also be taken as the hot spots of fire risk. It is worth noting that the spatial pattern of the three fire causes can be divided into two regions: the north and the south, among of which the hot spots are mainly concentrated in the north of China, such as Xinjiang, Neimenggu, Qinghai, Ningxia, and Gansu. This may be related with the difference of culture and living conditions between the north and the south regions. By comparison, there is less differentiation for other four fire causes. However, they still present spatial inequality. The distribution map of Spontaneous combustion has highlighted six provinces as being at risk, whose SEI is more than 1.5. These provinces are Liaoning, Neimenggu, Xinjiang, 
Shanxi(2), Fujian, and Beijing. The high-risk areas of arson are concentrated in Shanxi(2), Qinghai, Jilin, Neimenggu, Liaoning, Ningxia, and Xinjiang. There are no obvious regional characteristics for the electricity fires. The electricity fires are dispersal, and the high risk provinces include Xinjiang, Shanghai, Hunan, Neimengu, Chongqing, Fujian, and Beijing. Furthermore, there are significantly spatial distribution characteristics for the fire risk. An obvious conclusion is that the whole fire situation in the north of China is more serious than that of the south regions.

\section{Conclusions}

This paper focused on the spatial inequality of fire risk in China, and has identified the spatial distribution characteristics for different fire causes. The spatial inequality of fire risk is calculated based on $\mathrm{CV}$ and SEI. These results illustrate that the number of fires and the fire deaths show obviously spatial inequality, and the fire risk of different causes are associated with regions and provinces. Gini coefficients, $\mathrm{CV}$, and SEI, are capable of exploring these spatial distribution characteristics, and the spatial trends of fire risk. Based on the results of SEI, the risk distribution maps of fire occurrences, fire deaths, and fire causes are established by using ArcGIS. The maps can clearly demonstrate the spatial distribution of fire risk in each province, and simultaneously help the policy-makers to take more scientific and reasonable fire control strategies.

By comparing the three regions, the Gini coefficients of the number of fires in western region are more than 0.4 from 2003 to 2012, and the spatial inequality presents an intensified trend. Spatial analyses can explore the distribution of fire risk, but the underlying reasons of the difference between the regions should be revealed in the future. The fire causes are complex and various, and there are high levels of variation in spatial heterogeneity between these causes. The high-risk provinces of different fire causes can be identified based on the Lorenz curves and SEI. These provinces should be paid more attention. The maps will be very practical for the fire management and the fire rescue services. By mastering the fire risk information, the fire department can make specific countermeasures directly.

\section{Acknowledgements}

This study was supported by the National Natural Science Foundation of China (No. 71403254) and China Postdoctoral Science Foundation (2014M551823).

\section{References}

1. Hantson S, Pueyo S, Chuvieco E. Global fire size distribution is driven by human impact and climate. Global Ecology \& Biogeography 24(1)(2015)77-86.

2. Yang L, Chen H, Yang Y, et al. The Effect of Socioeconomic Factors on Fire in China. Journal of Fire Sciences 23(6) (2005)451-467.

3. Sufianto H, Green A R. Urban Fire Situation in Indonesia. Fire Technology 48(2) (2012)367-387

4. Nicklas Guldaker, Per-Olof Hallin. Spatio-temporal patterns of intentional fires, social stress and socioeconomic determinants: A case study of Malm02, Sweden. Fire Safety Journal 70(2014)71-80.

5. Corcoran J, Higgs G, Rohde D, et al. Investigating the association between weather conditions, calendar events and socio-economic patterns with trends in fire incidence: an Australian case study. Journal of Geographical Systems 13(2) (2011)193-226.

6. Fire Department of Ministry of Public Security, China fire services 2013. China Personnel Publishing House, Beijing (2013).

7. Lee, B; Song, J; Lee, M, Chung, J. The relationship between characteristics of forest fires and spatial patterns of forest types by the ecoregions of South Korea. Journal of Korean Forestry Society 97 (2008)1-9.

8. Bermudez, P. de Zea; Mendes, J.; Pereira, J. M. C. Spatial and temporal extremes of wildfire sizes in Portugal (1984-2004). International Journal of Wildland Fire 18(8) (2009) 983-991.

9. Qin, X; Li Z; Zhang ZH. Spatial and temporal distribution pattern of fires in china using modis data. 2010 IEEE International Symposium on Geoscience and Remote Sensing IGARSS 331-334, (2010).

10. Lu S; Mei P. et al. Fatality and influence factors in highcasualty fires: a correspondence analysis. Safety Science 50(2011)1019-1033.

11. Li, G.; Lu, S.; Zhang, H.et al. Correspondence analysis on exploring the association between fire causes and influence factors. Procedia Engineering 62(2013)581591.

12. Yang LZ; Zhou, and Deng, et al. Fire situation and fire characteristic analysis based on fire statistics of China. Fire Safety Journal 37(2002)785-802.

13. Clauset, Aaron; Wiegel, Frederik W. A Generalized Aggregation-Disintegration Model for the Frequency of Severe Terrorist Attacks. Journal of Conflict Resolution 54(1) (2010)179-197. 
14. Hipp, John R. Block, tract, and levels of aggregation: Neighborhood structure and crime and disorder as a case in point. American Sociological Review 72(5) (2007)659-680.

15. Ghiglino, C; Venditti, A. Wealth distribution and output fluctuations. Journal of Economic Theory 146(6) (2011) 2478-2509.

16. Viehweger, Adrian; Riffert, Till; Dhital, Bibek. The Gini coefficient: a methodological pilot study to assess fetal brain development employing postmortem diffusion MRI. Pediatric Radiology 44(10) (2014)1290-1301.

17. Piotr P. Graczyk. Gini Coefficient: A new way to express selectivity of kinase inhibitors against a family of kinases. Journal of Medicinal Chemistry 50(23) (2007)5773-5779.

18. Oliveira S, Pereira J M C, et al. Exploring the spatial patterns of fire density in Southern Europe using Geographically Weighted Regression. Applied Geography 51(2014)143-157.

19. Hassine N B. Economic Inequality in the Arab Region. World Development 66(C) (2015)532-556.

20. Frick J R, Goebel J, Schechtman E, et al. Using Analysis of Gini (ANOGI) for Detecting Whether Two Subsamples Represent the Same Universe The German Socio-Economic Panel Study (SOEP) Experience. Sociological Methods \& Research 34(4) (2006)427-468.

21. Liu BS. A Research on the Economic Regionalizing of China. China Soft Science 2(2008)81-90.
22. Abel-Schaad, D; Lopez-Saez, JA. Vegetation changes in relation to fire history and human activities at the Pena Negra mire (Bejar Range, Iberian Centre Mountain System, Spain) during the past 4,000 years. Vegetation History and Archaeobotany 22(3) (2013)199-214.

23. Chotikapanich D; Griffiths W. On calculation of the extended Gini coefficient. Review of Income and Wealth, 47(4) (2001)541-547.

24. Haidich, A. B.; Ioannidis, J. P. The Gini coefficient as a measure for understanding accrual inequalities in multicenter clinical studies. Journal of clinical epidemiology 57(4) (2004)341-348.

25. Liao T. F. Measuring and analyzing class inequality with the Gini index informed by model-based clustering. Sociological Methodology 36(1) (2006)201-224.

26. Kleiber, C., \& Kotz, S. A characterization of income distributions in terms of generalized Gini coefficients. Social Choice and Welfare 19(4)(2002)789-794

27. Atkinson, A. B., Bourguignon, F. Handbook of income distribution. Elsevier 1(2002)87-166.

28. Barr, N. The Economics of the Welfare State. 4th edition. Stanford University Press, USA, (2004).

29. Drckman, A; Jackson, T. Measuring resource inequalities: The concepts and methodology for an areabased Gini coefficient. Ecological economics 65(2) (2008)242-252.

30. Tziafetas, G. N. A Formula for the Gini Coefficient and its Decomposition. Biometrical Journal 31(8) (1989)961967. 